Check for updates

Cite this: Nanoscale Adv., 2019, 1, 3406

\title{
Anticancer drug delivery to cancer cells using alkyl amine-functionalized nanodiamond supraparticles $\dagger$
}

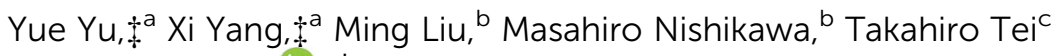 \\ and Eijiro Miyako (iD $\ddagger^{\star a}$
}

Nanocarriers have attracted increasing interest due to their potential applications in anticancer drug delivery. In particular, the ability of nanodiamonds (NDs) to spontaneously self-assemble into unique nano-structured architectures has been exploited in the development of nanocarriers. In this context, we synthesized functional supraparticles (SPs) by the self-assembly of alkyl amine-modified NDs for use in anticancer chemotherapy. The structural, physical, and physiological properties of these ND-SPs as well as their high biocompatibility were assessed using microscopic techniques and various characterization experiments. Finally, a model anticancer drug (CPT; camptothecin) was loaded into the ND-SPs to investigate their anticancer efficacy in vitro and in vivo. After incubation of CPT-loaded ND-SPs with cancer cells, a dramatic anticancer effect of ND-SPs was expressed, compared to CPT-loaded ordinary nanocarriers of polyethylene glycol-modified polymer micelles and conventional Intralipid® 20\% emulsions containing CPT. Our results demonstrated that ND-SPs may serve as a nanomedicine with significant therapeutic potential.

Received 23rd July 2019

Accepted 13th August 2019

DOI: 10.1039/c9na00453j

rsc.li/nanoscale-advances
Self-assembled materials have gained great attention with respect to the design of drug carriers and three-dimensional architectures due to their wide range of applications from controlled drug delivery and tissue engineering to nanoelectronics. ${ }^{\mathbf{8} 9}$ The ND surface possesses an assortment of functional groups, most of which are oxygenated and amino moieties, including carboxylic acid, lactone, ketone, ether, hydroxyl, and amino groups. These functional groups and/or the hydrophobic surface of NDs have mostly been used as a platform to conjugate with drug molecules through ionic, hydrogen, and hydrophobic interactions. ${ }^{\mathbf{1 0 - 1 2}}$ However, the use of these functional groups as a starting point and a driving force for the self-organization of built up nanoconstructs is still not sufficiently exploited. We believe that these self-assembled formulations of NDs can be further expanded as a modification concept to deliver drugs to site-specific targets. To explore such possibilities, we recently demonstrated that perfluorooctanoic acid (PFOA)-chemically functionalized NDs spontaneously transformed into well-dispersed and biocompatible supraparticle (SP) nanoclusters that could serve as effective drug carriers for cancer treatment. ${ }^{13}$ PFOA was used as a model inducer to achieve the SP transformation via strong hydrophobic interactions. However, fluorine compounds, including PFOA, have potential environmental and health risks due to their accumulation and retention in biological bodies. ${ }^{\mathbf{1 4 , 1 5}}$ Therefore, rational and new designs of ND-based SPs are desired for future clinical and biomedical applications. 
In this context, we used a variety of alkyl amine derivatives, which have relatively lower biological retention, as alternative materials for PFOA to explore their capacity to form ND-SPs. Different lengths of alkyl chains of amine derivatives were modified onto a carboxylic acid-functionalized ND via simple covalent modification into self-assembled SPs that have wellcontrolled particle sizes. These SPs were further modified with an anticancer drug to investigate the potential improvement of the drug efficacy in comparison to conventional nanocarriers composed of polyethylene glycol (PEG)-modified polymer micelles and an ordinary drug carrier of Intralipid® $20 \%$ emulsions.

\section{Experimental}

\section{ND-SP syntheses}

NDs (diameter $=4-5 \mathrm{~nm}$ ) were prepared via a detonation method. ${ }^{16-18}$ The synthesized NDs were purified with nitric acid and burned in a hydrogen gas atmosphere. Elemental analysis of the NDs (C (86.92\%), H (0.44\%), and N (2.29\%) contents) was performed using an organic elemental analyzer (Micro Corder JM10, J-Science Lab Co., Ltd, Kyoto, Japan). The purified NDs were dispersed in distilled water via bead milling (Sand Grinder LSG-4U, Aimex Co., Ltd, Tokyo, Japan). The ND aqueous suspension was then centrifuged to remove water-insoluble NDs. The supernatant (ND-ori) was used for further studies. A $1 \mathrm{ml}$ volume of ND-ori (ND concentration $=56 \mathrm{mg} \mathrm{ml}^{-1}$ ); $50 \mu \mathrm{l}$ of n-octylamine (Oct), oleylamine (Ole), or $10 \mathrm{mg}$ of dodecylamine (Dod) (FUJIFILM Wako Pure Chemical, Osaka, Japan); and $10 \mathrm{mg}$ of 1-ethyl-3-(3-dimethylaminopropyl) carbodiimide hydrochloride (WSC) (FUJIFILM Wako Pure Chemical) were dissolved in $9 \mathrm{ml}$ of 2-( $N$-morpholino)ethanesulfonic acid (MES) buffer ( $\mathrm{pH}$ 6.0, $100 \mathrm{mM}$ ) via bath sonication (power output, $80 \mathrm{~W}$; oscillation frequency, $40 \mathrm{kHz}$ ) (USD-2R, AS ONE, Osaka, Japan) for $5 \mathrm{~min}$. After vigorous stirring for another $1.5 \mathrm{~h}$ at room temperature, the mixture was centrifuged (MX-307, TOMY, Tokyo, Japan) and washed three times with Milli-Q water to remove unreacted chemicals. The resultant pellet was resuspended in $10 \mathrm{ml}$ of Milli-Q water via pulse-type sonication (VCX-600, Sonics, Danbury, CT, USA) for $10 \mathrm{~min}$. The obtained ND-SP product was used for the subsequent experiments. The concentration of NDs ( $c a .5 .6 \mathrm{mg} \mathrm{ml}^{-1}$ ) in the final product was estimated using an ultraviolet (UV)-visible (Vis)-near infrared (NIR) spectrophotometer (V-730 BIO, Jasco, Tokyo, Japan). The amounts of Oct (ca. $13 \mathrm{wt} \%$ ), Dod (ca. $17 \mathrm{wt} \%$ ), and Ole (ca. $35 \mathrm{wt} \%$ ) in the ND-SPs were analyzed via thermogravimetric analysis (TGA) (Q 500, TA Instruments, New Castle, DE, USA).

Camptothecin (CPT)-loaded Oct-ND-SP and Dod-ND-SP [CPT@Oct-ND-SP and CPT@Dod-ND-SP] complexes were prepared as follows. The washed Oct-ND-SP or Dod-ND-SP pellet was simply combined with $10 \mathrm{mg}$ of CPT (FUJIFILM Wako Pure Chemical) followed by pulse-sonication in $10 \mathrm{ml}$ of Milli-Q water for $10 \mathrm{~min}$. CPT@ND-ori was prepared by mixing $10 \mathrm{mg}$ of CPT, $1 \mathrm{ml}$ of ND-ori, and $9 \mathrm{ml}$ of Milli-Q water using a pulsetype sonicator for $10 \mathrm{~min}$. CPT@PEGMEM, CPT@F127, CPT@DSPE-PEG, and CPT@Intralipid® 20\% were obtained in the same manner except that the ND pellet was replaced with $56 \mathrm{mg}$ of poly(ethylene glycol) methyl ether methacrylate
(PEGMEM) (Sigma-Aldrich, St. Louis, MO, USA), $56 \mathrm{mg}$ of pluronic F127 (F127) (FUJIFILM Wako Pure Chemical), 56 mg of $N$-(aminopropyl polyethyleneglycol)carbamyl-distearoylphosphatidyl-ethanolamine (DSPE-PEG) (Sunbright DSPE020PA, Yuka Sangyo, Tokyo, Japan), or $1 \mathrm{ml}$ of Intralipid ${ }^{\circledR}$ 20\% (Sigma-Aldrich).

Boron dipyrromethene (BODIPY)-loaded Dod-ND-SP, PEGMEM, F127, and DSPE-PEG complexes [BODIPY@Dod-ND-SP, BODIPY@PEGMEM, BODIPY@F127, and BODIPY@DSPEPEG] were prepared in a similar manner to the preparation of the CPT-loaded nanocomplexes.

\section{Characterization of the ND-SPs}

The structure and morphology of the prepared ND-SPs were visualized using a high-resolution transmission electron microscope (TEM) (EM-002B, Topcon, Tokyo, Japan) at an accelerating voltage of $120 \mathrm{kV}$.

The hydrodynamic diameter of the ND-SPs was determined via dynamic light scattering (DLS) (Photal FPAR-1000, Otsuka Electronics, Osaka, Japan).

A UV-Vis-NIR spectrophotometer (V-730 BIO, Jasco, Tokyo, Japan) was used to measure the spectral profiles and concentrations of the ND-SPs and CPT complexes.

Fourier transform infrared (FTIR) spectroscopy (Spectrum One, PerkinElmer, Yokohama, Japan) analysis was carried out to identify the presence of alkyl chains on the ND surface.

\section{Cell culture and cytotoxicity evaluation}

U2OS bone osteosarcoma and TIG-3 and MRC5 normal human fibroblast cell lines were obtained from the Japanese Collection of Research Bioresources Cell Bank (Tokyo, Japan) and cultured in Dulbecco's Modified Eagle's Medium (Gibco, Grand Island, NY, USA) containing 10\% fetal bovine serum (Gibco), $2 \mathrm{mM}$ L-glutamine (Gibco), $1 \mathrm{mM}$ sodium pyruvate (Gibco), gentamycin (Gibco), penicillin-streptomycin (100 IU $\mathrm{ml}^{-1}$ ) (Gibco), and Hank's balanced salt solution (Life Technologies, Carlsbad, CA, USA). Cells were maintained at $37{ }^{\circ} \mathrm{C}$ in a humidified chamber at $5 \% \mathrm{CO}_{2}$.

Cell viability was assessed using a Cell Counting Kit (CCK)-8 (Dojindo Laboratories, Kumamoto, Japan) according to the manufacturer's instructions. Briefly, cells were seeded in a 96well plate $\left(5 \times 10^{3}\right.$ cells per well $)$ and allowed to attach overnight. Then, they were exposed to drugs or nanocomplexes as indicated. After washing with a fresh medium, the cells were incubated with the CCK-8 solution. A microplate reader (Infinite M200 PRO, Tecan, Männedorf, Switzerland) was used to read the absorbance at $450 \mathrm{~nm}$.

\section{In vitro releasing behavior of CPT from ND-SPs}

The CPT@Dod-ND-SP powder $(5.6 \mathrm{mg})$ was dispersed in $1 \mathrm{ml}$ of PBS buffer solution ( $\mathrm{pH} 7.4)$ at $37^{\circ} \mathrm{C}$ and stirred at $100 \mathrm{rpm}$. At determined time intervals, $50 \mu$ of the solution was collected and mixed with $200 \mu \mathrm{l}$ of dimethyl sulfoxide to completely dissolve the drugs. The mixture was then centrifuged at $15000 \mathrm{rpm}$ for $5 \mathrm{~min}$ to remove undissolved ND-SPs. The CPT concentration in the supernatant was finally analyzed using a UV-Vis-NIR spectrophotometer. 


\section{Live cell imaging}

U2OS cells were seeded in imaging dishes the night before treatment. The attached cells were incubated with BODIPYloaded conventional nanocarriers (PEGMEM, F127, and DSPEPEG) and BODIPY@Dod-ND-SPs for $3 \mathrm{~h}$ at $37{ }^{\circ} \mathrm{C}$, followed by nuclear staining with Hoechst $33342\left(1 \mu \mathrm{g} \mathrm{ml}{ }^{-1}\right.$; Thermo Fisher Scientific, Waltham, MA, USA) for $10 \mathrm{~min}$. After three washes with PBS, the cells maintained in the RPMI 1640 phenol red-free medium (Thermo Fisher Scientific) were subjected to live-cell imaging. The images were acquired with a fluorescence microscope (IX73, Olympus, Tokyo, Japan) equipped with a mirror unit (IX3-FGFPXL, Olympus) and an electron-multiplying charge-coupled device camera (DP80, Olympus).

\section{In vivo antitumor experiments}

All animal procedures were performed in accordance with the Guidelines for Care and Use of Laboratory Animals of National Institute of Advanced Industrial Science \& Technology (AIST) and experiments were approved by the Animal Ethics Committee of AIST. Female BALB/cAJc-nu/nu mice ( 6 weeks old, $18 \mathrm{~g}$ ) were bought from Japan SLC, Inc. (Shizuoka, Japan). Upon arrival, the mice were randomly separated into 3 groups $(n=4)$, and subcutaneously inoculated in the right flank with HT-29 cells $\left(5 \times 10^{5}\right.$ cells per site) for the construction of the tumor xenograft model. Intraperitoneal injections of $200 \mu \mathrm{l}$ of Dod-NDSPs (56 mg kg ${ }^{-1}$ ), CPT@ND-SPs (CPT, $3 \mathrm{mg} \mathrm{kg}^{-1}$; ND-SPs, $56 \mathrm{mg}$ $\mathrm{kg}^{-1}$ ) or PBS were performed every alternate day, when small tumor buds reach about $50 \mathrm{~mm}^{3}$. The overall health was monitored by body weight observation and the tumor size was calculated as $V=L \times W^{2} / 2$, where $L$ and $W$ are the length and width of the tumor, respectively.

\section{Blood tests}

The complete blood count and biochemical parameters were analyzed by Japan SLC, Inc. (Shizuoka, Japan) and Oriental Yeast Co., ltd. (Tokyo, Japan). Briefly, 10 week-old female BALB/ cSlc mice ( $n=5$; average weight $=21 \mathrm{~g}$; Japan SLC) were injected with $200 \mu \mathrm{l}$ of sterilized water containing ND-SPs (ND$\mathrm{SP}=1.12 \mathrm{mg} \mathrm{kg}^{-1}$ ) or $200 \mu \mathrm{l}$ of phosphate buffered saline (PBS) buffer via the tail vein. Blood samples were collected from the inferior vena cava of the mice after 4 weeks.

\section{Statistical analysis}

The results are presented as the mean \pm standard deviation of at least three independent experiments, with " $n$ " indicating the number of samples per group. Differences between the groups were evaluated using Student's $t$-test for two groups and two-way analysis of variance for multiple groups. $* * *$, and $* * *$ denote $p$ value is less than $0.05,0.01$, and 0.001 , respectively.

\section{Results and discussion}

\section{Synthesis and characterization of the ND-SPs}

To align the effective nanocarrier features of the SPs with alkyl amine molecules, which have a relatively lower

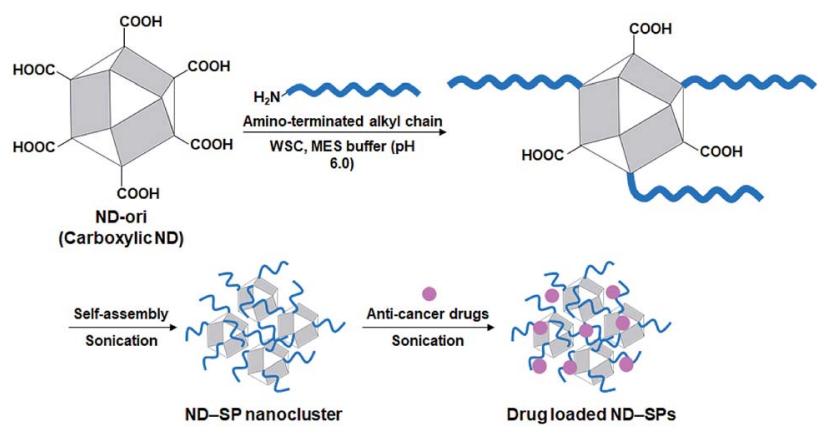

Fig. 1 Schematic of ND-SP synthesis. Crude ND-ori was functionalized via cross-linking between surface carboxylic groups and the amino groups of alkyl chains via a condensation reaction followed by sonication to promote the self-assembly of ND-SP nanoclusters.

bioaccumulation compared to fluorine compounds, carboxylic ND (ND-ori)-based SPs (ND-SPs) were designed and synthesized via a condensation reaction between the carboxylic group on the surface of ND-ori and the amino group of the primary alkyl amines, n-octylamine (Oct), dodecylamine (Dod), and oleylamine (Ole), using WSC in an MES buffer (pH 6.0) (Fig. 1). The alkyl chains of the amines immediately trigger the formation of SPs due to their hydrophobic properties. Sonication allows the transformation of the self-assembled SP nanoclusters and the encapsulation of the anticancer drugs into the assembly. The simple preparation of ND-SPs, in addition to the convenient drug loading method, is beneficial for various biomedical applications.

After the reaction, each of the ND-SP solutions, which have different alkyl chain lengths, showed unique colors and aspects (Fig. 2a). The turbidity of the samples became high due to surface scattering as the length of the alkyl chains increased. ${ }^{19}$ Indeed, the absorbances of the ND-SPs increased depending on their apparent turbidity (Fig. 2b). Of the solutions, the spectrum of Ole-ND-SPs showed the highest absorbance due to the suspension. Additionally, the TGA results revealed that approximately $13 \mathrm{wt} \%, 17 \mathrm{wt} \%$, and $35 \mathrm{wt} \%$ of Oct, Dod, and Ole, respectively, were modified onto the ND surface (Fig. 2c). The FTIR peaks, indicated with arrows in Fig. 2D, can be clearly assigned to $\mathrm{C}-\mathrm{H}$ stretch of the conjugated alkyl amines (Dod), further confirming the formation of ND-SPs.

Due to EPR effects, nano-scale (10-200 nm) particles are favorable for passively targeting tumors. ${ }^{1-3}$ Interestingly, according to the DLS measurements, the particle sizes of the prepared ND-SPs ( $c a$. 18-87 $\mathrm{nm}$ ) make them promising nanocarriers to express EPR effects (Fig. 3a). More interestingly, the diameters of ND-SPs can be easily controlled by changing the length of the alkyl chains. In fact, the DLS profiles of Oct-NDSPs, Dod-ND-SPs, and Ole-ND-SPs displayed well-defined size distributions with different average diameters of $18 \mathrm{~nm}, 41 \mathrm{~nm}$, $87 \mathrm{~nm}$, respectively (Fig. 3a). The solutions remained stable for at least one week with no obvious change in size. The hydrodynamic diameters of the ND-SPs were higher than those of NDori (ca. $15 \mathrm{~nm}$ ) due to the self-assembled nanocluster formations. The TEM observations clearly showed that the 
(a)

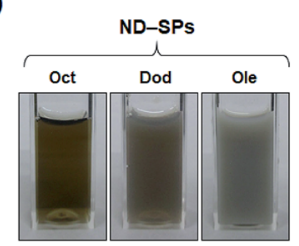

(c)

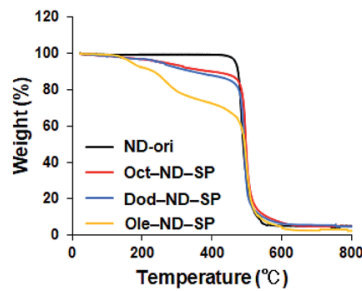

(b)

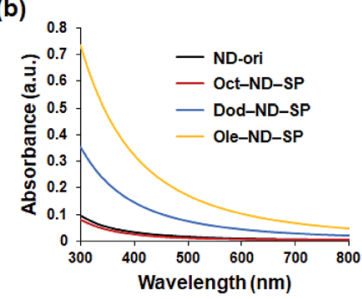

(d)

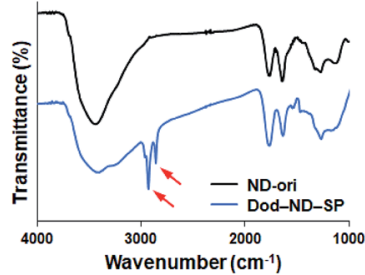

Fig. 2 (a) Photos of ND-SP aqueous solutions. The concentration of the NDs is $5.6 \mathrm{mg} \mathrm{ml}^{-1}$. (b) UV-Vis-NIR absorbance spectra of ND-SPs. The concentration of the NDs is $56 \mu \mathrm{g} \mathrm{ml}^{-1}$. (c) TGA curves of ND-ori and various ND-SPs. (d) FTIR spectra of ND-ori and Dod-ND-SPs, identifying the Dod moiety in the conjugates. Red arrows represent the evidence of $\mathrm{C}-\mathrm{H}$ stretch.

(a)

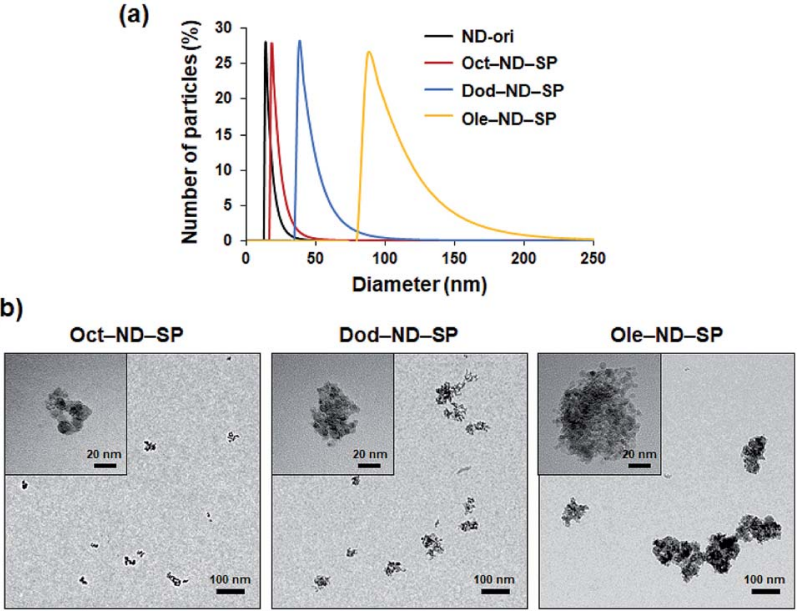

(b)

Fig. 3 (a) DLS hydrodynamic size distribution of the ND-SPs. (b) TEM images of the ND-SPs. High magnification images are shown in the upper left corners of the panels.

synthesized ND-SPs had cluster nanostructures based on the building blocks of ND-ori (Fig. 3b). The size of each ND-SP, estimated via TEM observations, depicted the same order of particle size as the DLS measurements. These results clearly indicate that alkyl amines can induce self-assembled SP formation of NDs via simple chemical functionalization.

Next, the cytotoxicity of the ND-SPs was investigated because this is a very important issue for future clinical and biomedical applications. CCK-8 assays were used to analyze the survival of the U2OS cells (Fig. 4a). The U2OS cells were pre-incubated with five different ND concentrations (ND $=0 \mu \mathrm{g} \mathrm{ml}^{-1}, 7 \mu \mathrm{g} \mathrm{ml} \mathrm{m}^{-1}, 14$ $\mu \mathrm{g} \mathrm{ml} \mathrm{m}^{-1}, 28 \mu \mathrm{g} \mathrm{ml}^{-1}$, and $56 \mu \mathrm{g} \mathrm{ml}^{-1}$ ) using the three types of ND-SPs. Over $98 \%$ of the cells were viable following the

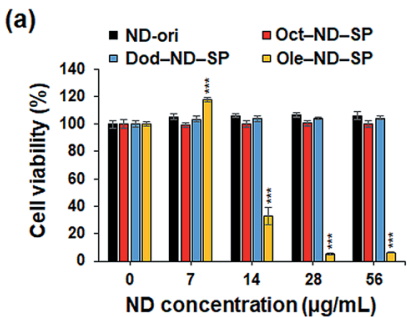

(b)

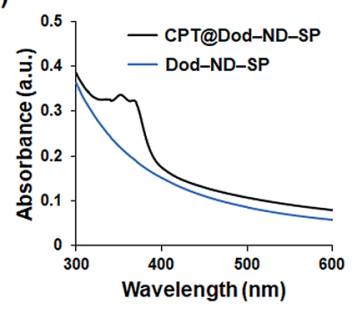

(c)

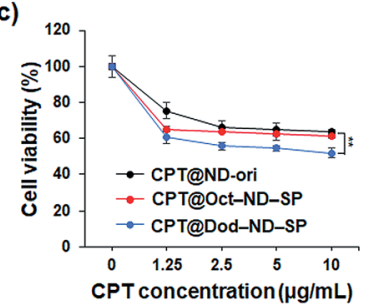

(d)

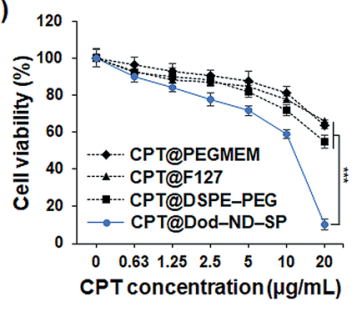

Fig. 4 (a) Cytotoxicity evaluation of various ND-SPs. The U2OS cell viability was assessed after $24 \mathrm{~h}$ of treatment. The data are presented as the mean $\pm \mathrm{SD}(n=5), * * * p<0.001$ (Student's $t$-test to $\left.0 \mu \mathrm{g} \mathrm{ml}^{-1}\right)$. (b) UV-Vis-NIR absorbance spectra of ND-SPs before (blue curve) and after (black curve) CPT incorporation. (c) Cell inhibitory effect of CPT@ND-ori, CPT@Oct-ND-SPs, and CPT@Dod-ND-SPs. The U2OS cancer cells were treated with the nanocomplexes for $24 \mathrm{~h}$. The data are presented as the mean \pm SD $(n=5), * * p<0.01$ (two-way ANOVA test). (d) Cytotoxicity evaluation of the CPT-loaded conventional nanocarriers (PEGMEM, F127, and DSPE-PEG) and CPT@Dod-ND-SPs in the U2OS cells after $24 \mathrm{~h}$ of treatment. The data are presented as the mean \pm SD $(n=6), * * * p<0.001$ (two-way ANOVA test).

treatment with Oct-ND-SPs or Dod-ND-SPs at all concentrations. Additionally, Ole-ND-SPs displayed a higher cytotoxicity of more than $14 \mu \mathrm{g} \mathrm{ml}^{-1}$ of $\mathrm{ND}$, likely due to the excess and stronger interaction between the cell surface and the longer alkyl chains of Ole, resulting in the denaturation of the cell membrane. Accordingly, Oct-ND-SPs and Dod-ND-SPs were used for further drug efficacy tests due to their low cytotoxicity.

The drug CPT has a wide range of antitumor effects on cancers. $^{20}$ CPT-based drugs are specific inhibitors of topoisomerase 1 , leading to the destruction of DNA, and are currently being used as useful chemotherapeutic agents in clinical antitumor treatments. The CPT molecules were encapsulated in the ND-SPs via simple sonication, as shown in Fig. 1. The UV-Vis-NIR spectra displayed characteristic peaks of CPT at $350 \mathrm{~nm}$ and $369 \mathrm{~nm}$ after encapsulation (Fig. 4b). To test the anticancer drug efficacy of the functionalized ND-SPs, we incubated the U2OS cells with CPT-loaded ND-SPs [CPT@OctND-SPs or CPT@Dod-ND-SPs] or CPT-loaded ND-ori (CPT@ND-ori) for $24 \mathrm{~h}$. After washing with a fresh growth medium, the cell viability was analyzed using a CCK-8 kit. CPT@Dod-ND-SPs showed the highest anticancer chemotherapeutic effect (ca. 49\%) at $10 \mu \mathrm{g} \mathrm{ml}^{-1}$ of CPT compared to the maximum drug efficacy values of the other materials [CPT@OctND-SPs at $\sim 39 \%$ and CPT@ND-ori at $\sim 36 \%$ ] (Fig. 4c). Interestingly, CPT@Dod-ND-SPs did not exhibit strong drug efficacy on TIG-3 and MRC5 normal fibroblast cell lines in comparison with U2OS osteosarcoma cells because of its potential targeting effect (Fig. S1a †). Besides, Dod-ND-SPs themselves do not have 
any cytotoxicity against these fibroblast cell lines (Fig. S1b $\dagger$ ). More surprisingly, the most dramatic decrease in cell viability (down to $10 \%$ ) was caused by increasing the CPT concentration to $20 \mu \mathrm{g} \mathrm{ml}{ }^{-1}$, where CPT@DOD-ND-SPs exhibited better anticancer efficacy than conventional nanocarriers such as PEGMEM-, F127-, and DSPE-PEG-based polymer micelles including CPT molecules. It is well known that carbon-based nanomaterials have high biological affinities against cells. ${ }^{21-23}$ Additionally, the lower drug efficacies of conventional nanocarriers are likely due to PEG moieties on their constructs that generally inhibit interactions with cells. ${ }^{24,25}$ To clarify the effective transmembrane permeation properties of ND-SPs, fluorescence live cell imaging was performed (Fig. 5). A hydrophobic fluorescent molecule (BODIPY) was co-assembled with ND-SPs via noncovalent hydrophobic interaction. Fluorescence microscopy showed that compared to PEGMEM-, F127-, and DSPE-PEGbased polymer micelles, Dod-ND-SPs were more efficiently internalized by the U2OS cells at $37{ }^{\circ} \mathrm{C}$. Furthermore, the efficacy of CPT suspended in Intralipid ${ }^{\circledR} 20 \%$ emulsion, which is one of the recommended drug carriers, was also compared with the anticancer effect of CPT@Dod-ND-SPs (Fig. S2 $\dagger$ ). Dod-NDSPs showed a stronger drug efficacy than Intralipid ${ }^{\circledR} 20 \%$. These results clearly indicate that drug-loaded functional NDSPs can effectively eliminate cancer cells.

The final goal of this research is to build a functional drug delivery system of ND-SPs. Herein, we have calculated the loading capacity and efficiency (Fig. S3a $\dagger$ ). In vitro release profiles of CPT molecules from Dod-ND-SPs were monitored in PBS buffer ( $\mathrm{pH}$ 7.4) (Fig. S3b $\dagger$ ). CPT molecules were rapidly released from Dod-ND-SPs over time, where it reached a plateau after about $24 \mathrm{~h}$, and were released up to $75 \%$ in PBS after $72 \mathrm{~h}$.

To demonstrate the feasibility of CPT-loaded ND-SPs for cancer treatment in vivo, the antitumor activity of CPT@DodND-SPs was evaluated using HT-29 tumor xenograft models. As shown in Fig. 6A, the tumor growth was strongly suppressed in the CPT@Dod-ND-SP treatment group as compared to the

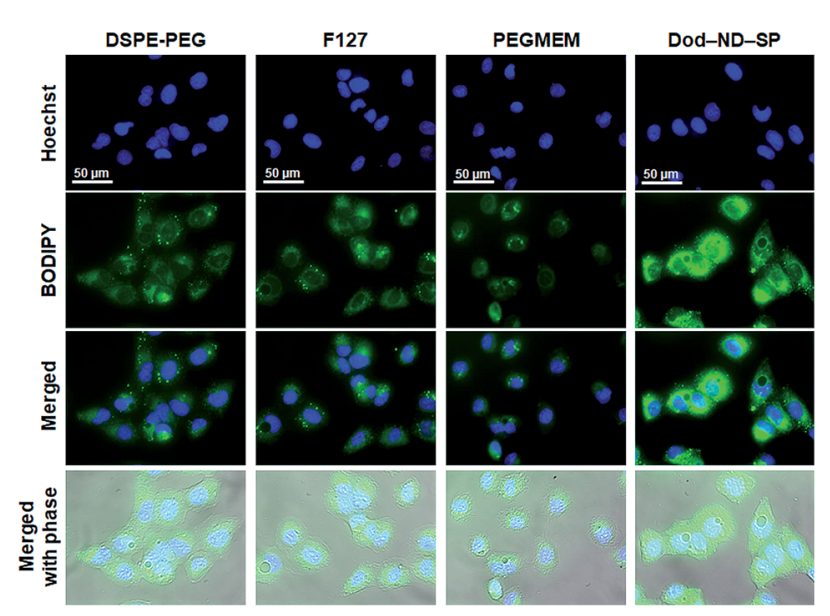

Fig. 5 Fluorescence micrographs of U2OS cells incubated with BODIPY@DSPE-PEG, BODIPY@F127, BODIPY@PEGMEM, and BODIPYaDod-ND-SPs for $3 \mathrm{~h}$. Concentrations of BODIPY and nanomaterials are $10 \mu \mathrm{g} \mathrm{ml}^{-1}$ and $56 \mu \mathrm{g} \mathrm{ml}^{-1}$, respectively. (a)

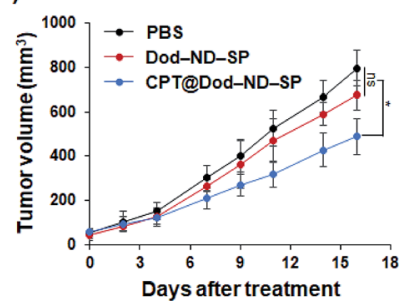

(b)

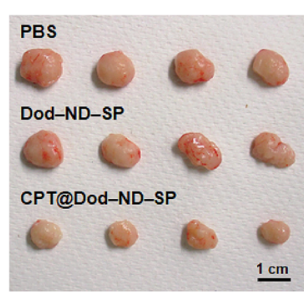

(c)

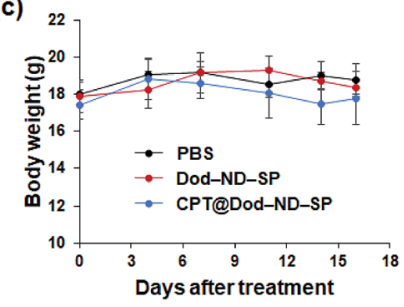

Fig. 6 In vivo anticancer effects. (a) Tumor growth curves of mice after intraperitoneal injection of CPTCDod-ND-SP (CPT, $3 \mathrm{mg} \mathrm{kg}^{-1}$,

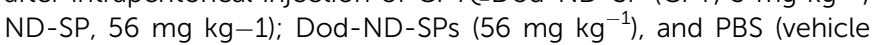
control). The data are presented as the mean $\pm \operatorname{SD}(n=4), * p<0.05$ (two-way ANOVA test). (b) Images of tumors isolated from mice at the end of the experiment. (c) Average body weight of different groups of mice during treatment.

PBS control, although Dod-ND-SPs alone didn't show significant difference. At the end of treatment, the tumor size of the mice receiving CPT@Dod-ND-SPs was remarkably smaller than those receiving PBS (Fig. 6B), suggesting that CPT@Dod-ND-SPs can retard tumor progression effectively. In addition, there was no significant loss of body weight in the mice (Fig. 6C), demonstrating that the systemic toxicity of CPT@Dod-ND-SPs is negligible.

To investigate the biocompatibility of ND-SPs further, mice were intravenously administered with sterilized water containing Dod-ND-SPs (200 $\mu \mathrm{l}$; Dod-ND-SPs, $\left.1.12 \mathrm{mg} \mathrm{kg}^{-1}\right)$ or $200 \mu \mathrm{l}$ of the PBS buffer for 4 weeks and subsequent blood tests were performed (Tables 1 and 2). The hematological and biochemical

Table 1 Hematological tests of mice injected with PBS or Dod-NDSPs after 4 weeks $^{a}$

\begin{tabular}{llccc}
\hline Entry & Unit & PBS $(n=5)$ & Dod-ND-SPs $(n=5)$ & $p$ value \\
\hline WBC & $\times 10^{2} \mu^{-1}$ & $85.3 \pm 13.4$ & $92.0 \pm 16.7$ & $>0.05$ \\
RBC & $\times 10^{4} \mu \mathrm{l}^{-1}$ & $923.8 \pm 21.9$ & $938.4 \pm 49.7$ & $>0.05$ \\
HGB & $\mathrm{g} \mathrm{dl}^{-1}$ & $13.9 \pm 0.6$ & $14.3 \pm 0.8$ & $>0.05$ \\
HCT & $\%$ & $41.4 \pm 1.9$ & $43.0 \pm 2.5$ & $>0.05$ \\
MCV & $\mathrm{fl}$ & $45.7 \pm 0.5$ & $45.9 \pm 0.5$ & $>0.05$ \\
MCH & $\mathrm{pg}$ & $15.3 \pm 0.2$ & $15.2 \pm 0.1$ & $>0.05$ \\
MCHC & $\mathrm{g} \mathrm{dl}^{-1}$ & $33.5 \pm 0.6$ & $33.2 \pm 0.5$ & $>0.05$ \\
PLT & $\times 10^{4} \mu \mathrm{l}^{-1}$ & $64.5 \pm 11.0$ & $70.1 \pm 2.7$ & $>0.05$
\end{tabular}

${ }^{a}$ Results represent the mean \pm standard deviation of five experiments. Statistical analyses were performed using Student's $t$-test. Abbreviations: WBC, white blood cells; RBC, red blood cells; HGB, hemoglobin; HCT, hematocrit; $\mathrm{MCV}$, mean cell volume; $\mathrm{MCH}$, mean cell hemoglobin; MCHC, mean cell hemoglobin concentration; and PLT, platelets. 
Table 2 Biochemical tests of mice injected with PBS or Dod-ND-SPs after 4 weeks $^{a}$

\begin{tabular}{llccl}
\hline & & & $p$ \\
Entry & Unit & PBS $(n=5)$ & Dod-ND-SPs $(n=5)$ & $\begin{array}{l}p \\
\text { value } v s . \text { PBS }\end{array}$ \\
\hline CRP & $\mathrm{mg} \mathrm{ml}^{-1}$ & $1.2 \pm 0.2$ & $0.9 \pm 0.2$ & $>0.05$ \\
TP & g dl $^{-1}$ & $3.9 \pm 0.2$ & $4.1 \pm 0.1$ & $>0.05$ \\
ALB & g dl $^{-1}$ & $2.6 \pm 0.2$ & $2.8 \pm 0.0$ & $>0.05$ \\
BUN & mg dl $^{-1}$ & $21.6 \pm 0.6$ & $23.5 \pm 3.1$ & $>0.05$ \\
CRE & mg dl $^{-1}$ & $0.13 \pm 0.02$ & $0.12 \pm 0.01$ & $>0.05$ \\
Na & meq. $^{-1}$ & $145.6 \pm 1.4$ & $148.6 \pm 0.5$ & $>0.05$ \\
K & meq. $^{-1}$ & $3.9 \pm 0.4$ & $3.4 \pm 0.2$ & $>0.05$ \\
Cl & meq. $^{-1}$ & $113.6 \pm 1.0$ & $117.0 \pm 0.9$ & $>0.05$ \\
AST & IU l $^{-1}$ & $59.4 \pm 11.0$ & $70.5 \pm 11.0$ & $>0.05$ \\
ALT & IU l $^{-1}$ & $31.2 \pm 5.0$ & $39.0 \pm 7.2$ & $>0.05$ \\
LDH & IU l $^{-1}$ & $230.0 \pm 41.0$ & $272.5 \pm 44.8$ & $>0.05$ \\
AMY & IU l $^{-1}$ & $1597.0 \pm 133.9$ & $1624.2 \pm 75.7$ & $>0.05$ \\
CK & IU l $^{-1}$ & $158.8 \pm 45.4$ & $166.4 \pm 59.1$ & $>0.05$
\end{tabular}

${ }^{a}$ Results represent the mean \pm standard deviation of five experiments. Statistical analyses were performed using Student's $t$-test. Abbreviations: CRP, C-reactive protein; TP, total protein; ALB, albumin; BUN, blood urea nitrogen; CRE, creatinine; AST, aspartate aminotransferase; ALT, alanine transferase; LDH, lactate dehydrogenase; AMY, amylase; and CK, creatine kinase.

parameters did not differ between the mice intravenously injected with Dod-ND-SPs and PBS, confirming the absence of an inflammatory response or systemic side effects and underscoring the biocompatibility of ND-SPs.

\section{Conclusions}

We demonstrated the spontaneous self-assembly of commercially available alkyl amine-modified NDs into unique geometrical architectures leading to the formation of SPs for the future treatment of osteosarcoma. We conducted a detailed investigation of SP formation for different lengths of alkyl chains of the primary amines. The structural, physical, and physiological properties of these ND-SPs were assessed using microscopic techniques and optical, thermal gravimetric, and cell tests. The sizes of the ND-SPs were easily controlled by changing the length of the alkyl chains of the amines. A model anticancer drug CPT was incorporated into these ND-SPs and conventional PEG-modified polymer micelles, and the ND-SPs showed good drug efficacy against U2OS bone osteosarcoma cells. In particular, CPT-loaded ND-SPs displayed the highest anticancer therapeutic effect. In vivo anti-tumor assays showed that CPT delivered by ND-SPs caused stronger tumor suppression of HT29 colorectal adenocarcinoma xenografts. These self-assembled ND-SPs open up possibilities for various applications and will be explored further for drug delivery. Compared to ordinary nanocarriers, ND-based materials generally possess the overwhelming superiorities of easy size control, robustness against chemicals and physical conditions, good thermal stability, and unique optical properties. ${ }^{\mathbf{2 6 , 2 7}}$ However, the investigation of NDSPs as drug carriers is still in its infancy. In this regard, developing ND-SPs is highly promising for various clinical and biological applications for cancer therapy.

\section{Conflicts of interest}

There are no conflicts to declare.

\section{Acknowledgements}

This work was supported by Daicel Corporation, a Japan Society for the Promotion of Science (JSPS) KAKENHI Grant-in-Aid for Scientific Research (A) [Grant number 19H00857], a JSPS KAKENHI Grant-in-Aid for Scientific Research (B) (no. 16H03834), and JSPS KAKENHI Fund for the Promotion of Joint International Research (Fostering Joint International Research) (no. 16KK0117).

\section{References}

1 Y. Matsumura and H. Maeda, Cancer Res., 1986, 46, 6387.

2 H. Maeda, J. Controlled Release, 2012, 164, 138.

3 R. Li, K. Zheng, C. Yuan, Z. Chen and M. Huang, Nanotheranostics, 2017, 1, 346.

4 D. Ho, C.-H. K. Wang and E. K. Chow, Sci. Adv., 2015, 1, e1500439.

5 J. Whitlow, S. Pacelli and A. Paul, J. Controlled Release, 2017, 261, 62.

6 Y. Einaga, Bull. Chem. Soc. Jpn., 2018, 91, 1752.

7 Y. Zhang, K. Y. Rhee, D. Hui and S.-J. Park, Composites, Part $B, 2018,143,19$.

8 M. Komiyama, K. Yoshimoto, M. Sisido and K. Ariga, Bull. Chem. Soc. Jpn., 2017, 90, 967.

9 J. Shi, P. W. Kantoff, R. Wooster and O. C. Farokhzad, Nat. Rev. Cancer, 2017, 17, 20.

10 E. K. Chow, X. Q. Zhang, M. Chen, R. Lam, E. Robinson, H. Huang, D. Schaffer, E. Osawa, A. Goga and D. Ho, Sci. Transl. Med., 2011, 3, 73ra21.

11 X. Wang, X. C. Low, W. Hou, L. N. Abdullah, T. B. Toh, M. M. A. Rashid, D. Ho and E. K. Chow, ACS Nano, 2014, 8, 12151.

12 B. Guan, F. Zou and J. Zhi, Small, 2010, 6, 1514.

13 Y. Yu, M. Nishikawa, M. Liu, T. Tei, S. C. Kaul, R. Wadhawa, M. Zhang, J. Takahashi and E. Miyako, Nanoscale, 2018, 10, 8969.

14 R. Seals, S. M. Bartell and K. Steenland, Environ. Health Perspect., 2011, 119, 119.

15 R. Ghisi, T. Vamerali and S. Manzetti, Environ. Res., 2019, 169, 326.

16 V. V. Danilenko, Combust., Explos. Shock Waves, 2005, 41, 577.

17 V. Y. Dolmatov, J. Superhard Mater., 2008, 30, 233.

18 V. Y. Dolmatov, V. Myllymäki and A. Vehanen, J. Superhard Mater., 2013, 35, 143.

19 R. Jethra, ISA Trans., 1993, 32, 397.

20 E. Martino, S. Della Volpe, E. Terribile, E. Benetti, M. Sakaj, A. Centamore, A. Sala and S. Collina, Bioorg. Med. Chem. Lett., 2018, 27, 701.

21 A. Bianco, K. Kostarelos and M. Prato, Chem. Commun., 2011, 47, 10182. 
22 A. C. Tripathi, S. A. Saraf and S. K. Saraf, Materials, 2015, 8, 25 I. Ozer, A. Tomak, H. M. Zareie, Y. Baran and V. Bulmus, 3068.

Biomacromolecules, 2017, 18, 2699.

23 E. J. Wallace and M. S. Sansom, Nano Lett., 2008, 8, 2751. 26 Nanodiamond, ed. O. A. Williams, RSC, 2014.

24 L. Xu, J. Yang, B. Xue, C. Zhang, L. Shi, C. Wu, Y. Su, X. Jin, 27 Nanodiamonds, ed. J.-C. Arnault, Elsevier, 2017. Y. Liu and X. Zhu, Biomaterials, 2017, 147, 1. 